\title{
Can optical coherence tomography replace fluorescein angiography in detection of ischemic diabetic maculopathy?
}

\author{
Diana Anna Dmuchowska • Pawel Krasnicki • \\ Zofia Mariak
}

Received: 23 May 2013 /Revised: 3 October 2013 / Accepted: 4 November 2013 / Published online: 29 November 2013

(C) The Author(s) 2013. This article is published with open access at Springerlink.com

\begin{abstract}
Background We studied the association between (1) the retinal thickness, volume and structure assessed by optical coherence tomography (OCT), and (2) vascular changes around the fovea in FA to define the role of these methods in the detection of ischemic diabetic maculopathy.

Methods This retrospective observational study included 51 eyes of 51 patients with severe non-proliferative and proliferative diabetic retinopathy, considering that they present advanced ischemic retinal changes. Based on the clinical examination and presence of leakage on FA, patients with clinically significant macular edema were excluded.

On FA, foveal avascular zone (FAZ) corresponds to capillary loss and reflects ischemic processes. Its outline and size were assessed according to the ETDRS standards. Patients were divided in two groups according to the severity of damage of the FAZ outline: $\leq$ grade $2(n=28)$ and $\geq$ grade 3 $(n=23)$.

As we expected ischemia-related damage, SD-OCT images were evaluated for retinal structure, volume, total thickness with division into the outer and inner retina (to assess the influence of two, non-overlapping blood sources) and ganglion cell layer thickness.

Results The comparisons revealed that at least currently with described methods:

1. There is no significant association between FAZ outline and retinal volume, total thickness, and thickness of the outer and inner retina and ganglion cell layer.
\end{abstract}

The authors have full control of all primary data, and they agree to allow Graefe's Archive for Clinical and Experimental Ophthalmology to review their data if requested.

D. A. Dmuchowska $(\bowtie) \cdot$ P. Krasnicki $\cdot$ Z. Mariak

Department of Ophthalmology, University Teaching Hospital of Bialystok, 24a M. Sklodowskiej-Curie St., 15-276 Bialystok, Poland e-mail: diana_anka@op.pl
2. There is no significant association between FAZ outline and following retinal structure characteristics: continuity of the external limiting membrane and inner segment/outer segment junction, and identification of the inner/outer retina boundary.

3. The identification of ganglion cell layer boundaries was significantly more difficult in more advanced FAZ outline grades.

4. FAZ size is not correlated with the retinal thickness and volume.

Conclusions In patients with severe non-proliferative and proliferative diabetic retinopathy, it is not possible to predict FAZ outline/size based solely on the measurements assessing volume and thickness or retinal structure evaluation on OCT. Consequently, at present OCT cannot replace FA in the detection of ischemic diabetic maculopathy.

Keywords Ischemic diabetic maculopathy $\cdot$ Retinal thickness $\cdot$ Foveal avascular zone outline $\cdot$ OCT $\cdot$ Fluorescein angiography

\section{Introduction}

Fluorescein angiography (FA) plays an important role in diabetic retinopathy (DR) staging and identification of fluorescein leakage sources for the laser treatment of the macula. At the present time, optical coherence tomography (OCT) has become popular in diagnosing and monitoring of diabetic macular edema and its laser, medical, and surgical treatment. In order to define the role of the two imaging methods in the detection of ischemic diabetic maculopathy without clinically significant macular edema (CSME), we studied the association between (1) the retinal thickness, volume, and structure assessed by OCT, and (2) vascular changes around the fovea in FA. 
There are two non-overlapping sources of blood supply to the retina. The outer retina is supplied via diffusion from the choriocapillaris. The inner retina is supplied by the central retinal artery. Macular vasculature consists of two capillary plexuses. Superficial capillary plexus lies in the nerve fiber layer or ganglion cell layer, while deep capillary plexus is located within the inner nuclear layer. There are no superficial capillaries in the foveola itself and in the immediate parafoveal retina, making the fovea dependent on the blood supply from the choriocapillaris. This area represents the foveal avascular zone (FAZ), best assessed in early phase of FA. Pathologic conditions that feature retinal capillary dropout, such as diabetes, may show an enlargement and irregular margins of the FAZ [1]. On the other hand, diabetes also leads to reduced choroidal circulation and thickness [2-4], which might lead to hypoxia of the outer retina. Expecting damage due to ischemia, we evaluated retinal volume, thickness, and structure. In addition to total retinal thickness measurement, we also divided the retinal thickness into the outer and inner retina in order to assess the influence of reduced blood supply from these two sources separately. FAZ is of a larger size in diabetic patients in comparison to controls [5-8] and increases as DR advances [5-8]; however, even in healthy subjects FAZ dimensions range from 0 to $1200 \mu \mathrm{m}$ [1]. Therefore, our study was mainly focused on FAZ outline, which corresponds to capillary loss and reflects ischemic processes, and seems to be a more reliable parameter with regards to representing the degree of potential blood supply insufficiency to the inner retina as compared to FAZ size. Conrath et al. assessed FAZ outline and size in patients with DR without CSME, and suggested employing FAZ outline as either complementary or in place of FAZ size in relevant studies [8].

Retinal thickness is generally higher in diabetic patients in comparison to controls [9-12], and higher in patients with DR than in those without signs of DR [9-11].

A number of papers on DR compare the retinal thickness and structure on OCT with AF images; however, they mainly deal with diabetic maculopathy with dye leakage [9, 13-15]. In contrast, we focused on diabetic maculopathy without dye leakage, and evaluated the association with FAZ outline. Several recent reports have suggested [16-18] that it is not possible to detect ischemic diabetic maculopathy based on OCT findings. Conversely, Byeon et al., who assessed foveal ganglion cell layer damage in ischemic diabetic maculopathy, postulated that OCT provides objective results and seems to be a good noninvasive substitute for FA [19]. Moreover, Van Dijk et al. proved that the duration of diabetes is significantly correlated with ganglion cell layer (GCL) thickness [20]. As the data from the literature is inconclusive, the present study addressed the question whether it is possible to predict the foveal avascular zone (FAZ) outline and size, and thus detect ischemic maculopathy, based on the retinal thickness measurement and retinal structure evaluation on OCT.

\section{Materials and methods}

This retrospective observational cross-sectional study included 51 eyes of 51 patients. The characteristics are summarized in Table 1 . The study was performed according to the Declaration of Helsinki, and was approved by the ethics committee at the Medical University of Bialystok. All patients gave written informed consent prior to all examinations.

We performed visual acuity testing, tonometry, and slitlamp biomicroscopy. DR and CSME staging were defined according to the Early Treatment Diabetic Retinopathy Study Research Group (ETDRS) [21-23]. We included patients with severe non-proliferative diabetic retinopathy (NPDR) and proliferative diabetic retinopathy (PDR), as they present advanced ischemic retinal changes. Moreover, this group is often omitted in studies, which tend to focus on earlier stages of DR.

Fluorescein angiography (Kowa Co. Ltd., Tokyo, Japan) was performed according to the standard procedure, and used to assess the severity of DR, characterize the FAZ according to ETDRS standards [24], and exclude CSME defined as dye leakage within $500 \mu \mathrm{m}$ radius from the fovea or $1,500 \mu \mathrm{m}$ radius in cases with hard exudates in this area. An early phase frame (after 20-30 seconds) and a late frame (after 3-5 minutes) were selected for further analysis. Severity of perifoveal capillary occlusion was characterized by the outline and size of the FAZ. Examples of FAZ outlines are shown on Fig. 1.

FAZ was manually outlined. The following dimensions were measured: horizontal and vertical linear dimensions passing through the presumed foveal center, as well as the greatest and smallest linear dimensions passing through the presumed foveal center in any meridian (Fig. 2). Then the arithmetic mean was calculated from the horizontal and vertical linear dimensions.

CSME was excluded based on clinical examination and presence of leakage on fluorescein angiograms. Patients were divided into two groups according to the severity of damage to the FAZ outline: $\leq$ grade $2(n=28)$ and $\geq$ grade $3(n=23)$.

SD-OCT was performed using TOPCON 3D OCT-1000 (Topcon Medical Systems Inc., Oakland, CA, USA) with axial resolution of $6 \mu \mathrm{m}$. Retinal thickness, volume, structure, and vitreomacular interface were assessed. Retinal thickness, defined as the distance between the inner retinal surface and retinal pigment epithelium, was measured automatically. The averaged thickness in the nine ETDRS fields was analyzed [24]. Next, the outer (from the retinal pigment epithelium to and including the outer plexiform layer) and inner retinal thicknesses [from and including the inner nuclear layer to the inner retinal surface (temporally)/nerve fiber layer (nasally)] was measured manually as demonstrated in Fig. 3. Ganglion cell layer, defined as the distance between the inner plexiform layer and nerve fiber layer, was separately assessed.

Retinal structure evaluation employing a scan traversing horizontally through the presumed foveal center included 
Table 1 Baseline characteristics of patients enrolled into the study

$\mathrm{FAZ}=$ foveal avascular zone; NPDR $=$ non-proliferative diabetic retinopathy;

$\mathrm{PDR}=$ proliferative diabetic retinopathy

\begin{tabular}{lllll}
\hline & All & FAZ outline grade 0, 1, & FAZ outline grade 3, 4 & $P$ \\
\hline$N$ & 51 & 28 & 23 & \\
Age (years) & $55.1 \pm 13.8$ & $59.4 \pm 10.2$ & $49.7 \pm 15.9$ & 0.019 \\
Female/male & $30 / 21$ & $18 / 10$ & $12 / 11$ & 0.408 \\
Severe NPDR/PDR & $16 / 35$ & $11 / 17$ & $5 / 18$ & 0.232 \\
\hline
\end{tabular}

continuity of the external limiting membrane and inner segment/outer segment junction (within the distance of $500 \mu \mathrm{m}$ nasally and temporally from the fovea), identification of a boundary between the inner and outer retina, and identification of ganglion cell layer boundaries.

The following exclusion criteria were met: presence of CSME (based on slit-lamp biomicroscopy and FA), prior posterior segment surgery or intravitreal injections, macular laser photocoagulation, ametropia $\geq 3.0$ diopters, macular changes resulting from other ocular diseases, and insufficient quality of fluorescein angiograms or OCT images. Moreover, patients with cilioretinal artery detected on fluorescein angiograms were excluded. Cilioretinal artery may be present in $20 \%$ of normal eyes; its significance is derived from the fact that in such a case the inner layer of the macula is dually supplied by the choroidal and retinal circulations.

Statistical analyses were performed with the SPSS 16.0 (SPSS Inc., Chicago, IL, USA). Data were presented as means \pm standard deviations or medians (min-max). Independent samples $t$-test for continuous data with normal distributions, Mann-Whitney U test for continuous data with non-normal distributions, and chi-square analysis for categorical variables

Fig. 1 Examples of fluorescein a grade $0=$ normal; $\mathbf{b}$ grade visible irregularities not definitely circumference; $\mathbf{d}$ grade $3=$ outline definitely destroyed for one half or more of the original destroyed; grade $8=$ cannot grade (according to the ETDRS report Angiograms with grade 8 of FAZ angiograms presenting FAZ outlines of study patients: $1=$ questionable, outline not smoothly round or oval, but abnormal; c grade $2=$ outline definitely destroyed in less than one half the original circumference, but some remnants remain; e grade $4=$ capillary outline completely number 11: "Classification of diabetic retinopathy from fluorescein angiograms" [24]). outline were excluded from the study

\section{Fig. 1 Examples of fluorescein} (1)

were used to test the difference between the groups. Correlation was performed with Spearmann's test. $P$ value less than 0.05 was considered statistically significant.

\section{Results}

Table 1 shows the clinical characteristics of 51 eyes of 51 patients with severe NPDR and PDR. None of the patients presented with CSME. Patients were divided into two groups according to their FAZ outline: $\leq$ grade $2(n=28)$ and $\geq$ grade $3(n=23)$. The patients in the second group were younger. There were no statistically significant differences in sex or diabetic retinopathy distribution between the groups.

Comparison of retinal thickness measurements and total volume values in relation to FAZ outline is presented in Table 2. In the case of FAZ outline $\leq$ grade 2, the retina was generally thicker and possessed a larger volume; however, the differences were not statistically significant. This tendency was not valid for the ganglion cell layer thickness.

The following median (min-max) FAZ dimensions were obtained: horizontal $1160 \mu \mathrm{m}$ (532-6539), vertical $976 \mu \mathrm{m}$

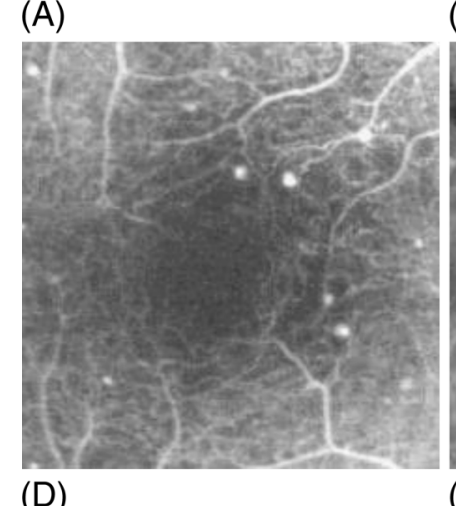

(D)

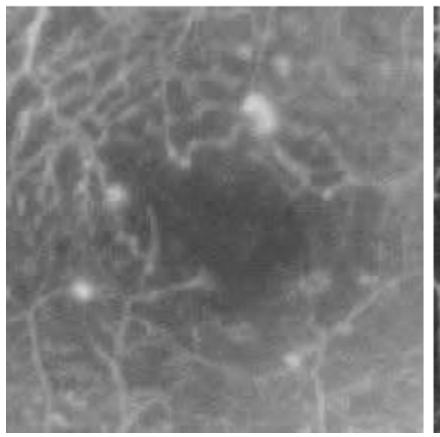

(A)
(B)

(C)

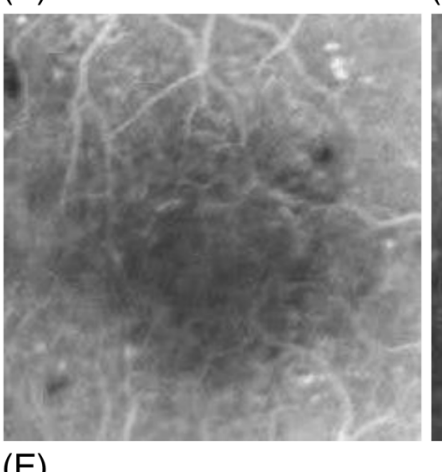

(E)
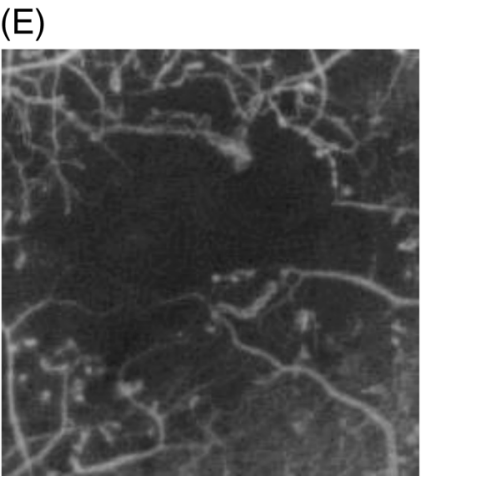

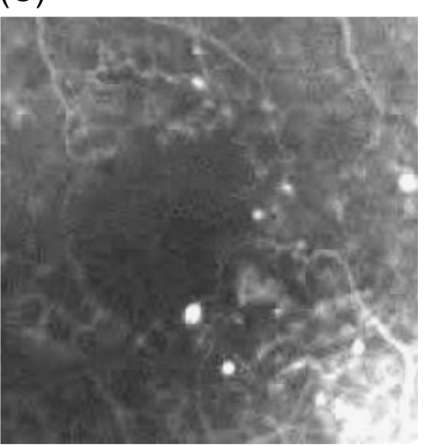




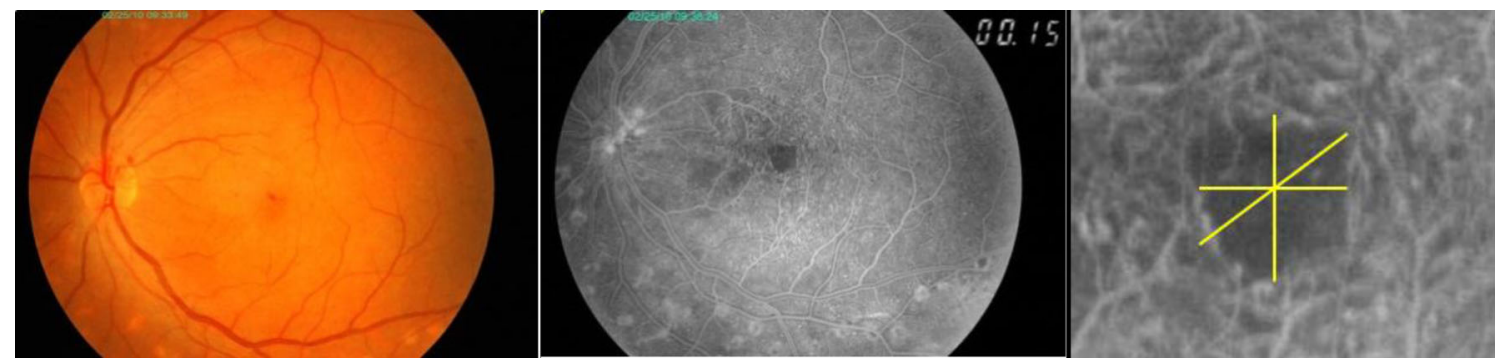

Fig. 2 Sample color fundus photograph and early-phase angiogram. Magnified photo of FAZ presents measurements of horizontal, vertical, and largest linear dimensions

(391-4366), mean $1102 \mu \mathrm{m}(461-5452)$; greatest $1261 \mu \mathrm{m}$ (537-6539) and smallest $867 \mu \mathrm{m}$ (360-2674). The FAZ dimensions were statistically significantly greater in the group with more destroyed FAZ outline $(p<0.001)$.

Correlation of retinal thickness and volume with FAZ size proved not significant (Table 3).

The comparison of the retinal structure between group 1 (FAZ outline $\leq$ grade 2 ) and group 2 (FAZ outline $\geq$ grade 3 ) did not reveal any significant differences. The assessed features included: continuity of the external limiting membrane $(p=0.769)$, and inner segment/outer segment junction $(p=0.120)$. Identification of the boundary between the inner and outer retina was not possible in one out of 28 patients in group 1 (FAZ outline $\leq$ grade 2 ) and three out of 23 subjects in group 2 (FAZ outline $\geq$ grade 3$)(p=0.211)$. The identification of ganglion cell layer boundaries was not possible in $29 \%$ of patients in group 1 (FAZ outline $\leq$ grade 2 ) and $65 \%$ of subjects in group 2 (FAZ outline $\geq$ grade 3$)(p=0.009)$.

\section{Discussion}

The study has demonstrated that, at least at present with described methods, in patients with severe NPDR and PDR without CSME:

(1) It is not possible to predict the FAZ outline/size based solely on the measurement of thickness and retinal structure evaluation in OCT.

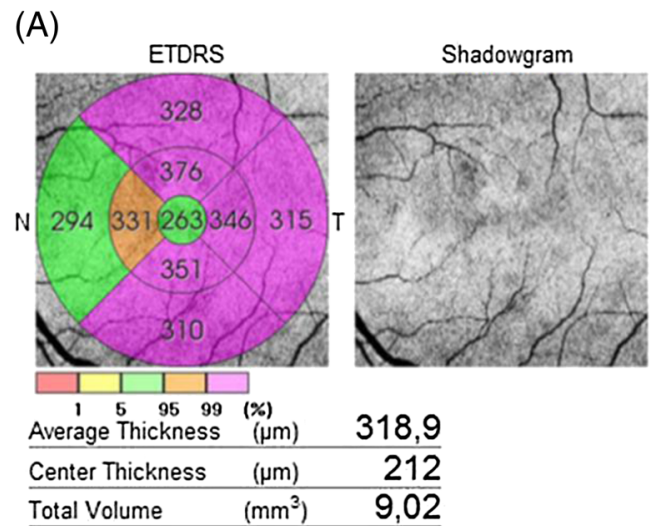

(B)

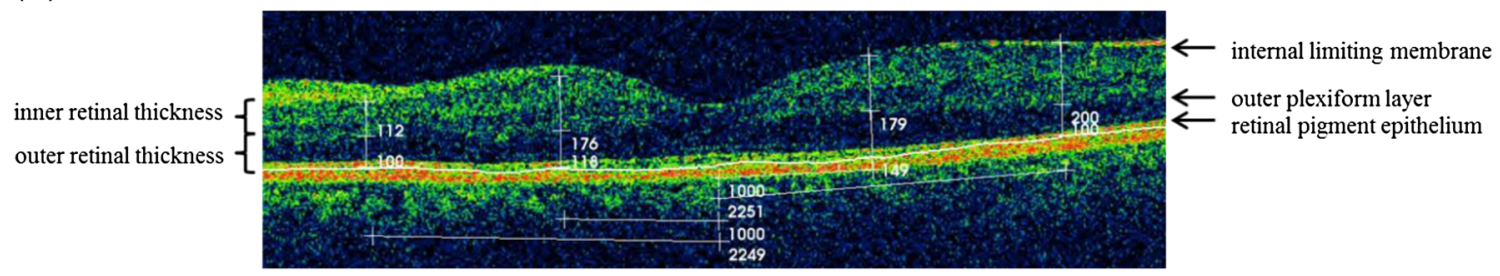

Fig. 3 Sample OCT image. a The total volume and averaged thickness in the nine ETDRS fields were measured automatically: central macular (central field within a $500 \mu \mathrm{m}$ radius), four parafoveal (within a 500 $1500 \mu \mathrm{m}$ radius) and four perifoveal subfields (within a $1500-3000 \mu \mathrm{m}$ radius) [24]. b The outer and inner retinal thickness was measured manually on the horizontal scans in the middle of the parafoveal region
(1000 $\mu \mathrm{m}$ from the fovea) and in the middle of the perifoveal region (2250 $\mu \mathrm{m}$ from the fovea) nasally and temporally. The outer thickness was defined as the distance between retinal pigment epithelium to and including the outer plexiform layer, while the inner thickness corresponded to the area from and including the inner nuclear layer and the inner retinal surface (temporally)/nerve fiber layer (nasally) 
Table 2 Comparisons of retinal volume and thickness in relation to FAZ outline

\begin{tabular}{|c|c|c|c|c|c|}
\hline & & All & FAZ outline grade $0,1,2$ & FAZ outline grade 3,4 & $P$ \\
\hline \multirow[t]{9}{*}{ Total retinal thickness $(\mu \mathrm{m})^{*}$} & CMT & $236.4 \pm 30.1$ & $241.0 \pm 28.9$ & $233.3 \pm 31.9$ & 0.449 \\
\hline & paraS & $298.3 \pm 32.5$ & $303.5 \pm 36.7$ & $296.5 \pm 28.9$ & 0.453 \\
\hline & paraT & $281.5 \pm 36.1$ & $291.0 \pm 32.3$ & $273.6 \pm 41.6$ & 0.179 \\
\hline & paraI & $287.6 \pm 31.7$ & $292.3 \pm 25.2$ & $284.1 \pm 37.2$ & 0.467 \\
\hline & paraN & $296.8 \pm 28.2$ & $300.5 \pm 26.6$ & $294.6 \pm 32.4$ & 0.526 \\
\hline & periS & $272.1 \pm 24.4$ & $273.4 \pm 25.1$ & $270.6 \pm 24.0$ & 0.689 \\
\hline & periT & $257.6 \pm 30.8$ & $263.3 \pm 24.9$ & $256.0 \pm 40.2$ & 0.189 \\
\hline & periI & $264.1 \pm 23.7$ & $261.8 \pm 20.0$ & $266.7 \pm 26.6$ & 0.465 \\
\hline & periN & $284.7 \pm 25.7$ & $283.6 \pm 20.5$ & $286.2 \pm 31.3$ & 0.734 \\
\hline \multirow[t]{4}{*}{ Outer retinal thickness $(\mu \mathrm{m})^{* *}$} & periT & $112.9 \pm 21.1$ & $116.5 \pm 19.2$ & $107.2 \pm 24.0$ & 0.138 \\
\hline & paraT & $126.6 \pm 21.7$ & $129.0 \pm 23.7$ & $121.6 \pm 20.3$ & 0.306 \\
\hline & paraN & $117.7 \pm 20.5$ & $115.6 \pm 20.6$ & $119.6 \pm 21.8$ & 0.398 \\
\hline & periN & $103.0 \pm 15.4$ & $99.0 \pm 12.1$ & $110.1 \pm 19.5$ & 0.011 \\
\hline \multirow[t]{4}{*}{ Inner retinal thickness $(\mu \mathrm{m})^{* *}$} & periT & $142.4 \pm 34.2$ & $146.0 \pm 31.8$ & $140.3 \pm 43.1$ & 0.511 \\
\hline & paraT & $157.6 \pm 25.6$ & $161.9 \pm 27.9$ & $154.9 \pm 25.7$ & 0.278 \\
\hline & paraN & $159.6 \pm 30.6$ & $169.1 \pm 29.6$ & $146.7 \pm 27.7$ & 0.011 \\
\hline & periN & $130.3 \pm 18.1$ & $132.8 \pm 18.4$ & $127.1 \pm 17.5$ & 0.291 \\
\hline \multirow[t]{4}{*}{ Ganglion cell layer thickness $(\mu \mathrm{m})^{* * *}$} & periT & $83.7 \pm 14.7$ & $81.0 \pm 15.9$ & $90.1 \pm 9.0$ & 0.202 \\
\hline & paraT & $89.2 \pm 12.6$ & $92.1 \pm 11.2$ & $84.0 \pm 13.8$ & 0.132 \\
\hline & paraN & $87.1 \pm 15.5$ & $86.5 \pm 15.6$ & $88.9 \pm 16.1$ & 0.839 \\
\hline & periN & $69.1 \pm 13.2$ & $65.3 \pm 10.4$ & $76.3 \pm 17.4$ & 0.078 \\
\hline Total volume $\left(\mathrm{mm}^{3}\right)^{*}$ & & $7.74 \pm 0.64$ & $7.80 \pm 0.61$ & $7.72 \pm 0.77$ & 0.599 \\
\hline
\end{tabular}

$\mathrm{FAZ}=$ foveal avascular zone; $\mathrm{CMT}=$ central macular thickness; peri $=$ perifoveal; para $=$ parafoveal; $\mathrm{S}=$ superior; $\mathrm{T}=$ temporal; $\mathrm{I}=$ inferior; $\mathrm{N}=$ nasal; $*_{n}=51 ; * *_{n}=47 ; * * * n=28$

(2) There is no significant association between FAZ outline and: retinal volume, total thickness, and thickness of the outer and inner retina and ganglion cell layer.

(3) There is no significant association between FAZ outline and the following retinal structural characteristics: continuity of the external limiting membrane and inner segment/outer segment junction, and identification of the inner/outer retina boundary.

(4) Identification of ganglion cell layer boundaries was more difficult in more advanced FAZ outline grades.

(5) FAZ size is not correlated with the retinal thickness and volume.

(6) FAZ size is significantly larger in more advanced FAZ outline grades.

We did not find significant thickness differences, and consequently volume, in association with the severity of FAZ damage. The ischemic changes could theoretically lead to atrophy and result in reduced thickness. On the other hand, these changes might be compensated by edematous enlargement of glial cells, as explained by Yanoff et al. and Fine et al. $[25,26]$. Another explanation might be that most of the cells in the area of perifoveal hypo- and nonperfusion survive, though in a semi-starved state. This hypothesis cannot be proved based on the currently available OCT images, as their resolution does not allow us to count individual cells. The same mechanisms could account for the lack of difference in the inner retinal thickness in relation to FAZ damage. The findings of Sanchez-Tocino et al. and Lattanzio et al. [11, 12] support our hypothesis. They showed that there were no significant differences in average thickness in any area between NPDR and PDR without CSME. Similarly, Ozdek et al., who evaluated patients with diabetic ischemic maculopathy as well as with CSME, did not find a significant difference in foveal thickness between the eyes with NPDR and PDR and in eyes with and without macular ischemia [17]. Furthermore, it is known that FA evaluates the superficial capillary plexus. In cases where superficial capillary dropout is present, deep capillary plexus still may be intact and vice versa. Although FAZ outline was not destroyed (grade $\leq 1$ ) in $14(27.5 \%)$ of our patients, we found evidence of ischemic changes within the retina as reflected by severe NPDR and PDR. Diabetes causes reduced choroidal circulation [27, 28] and thickness [2-4], which might lead to hypoxia of the outer retina. We did not assess the choroidal thickness and flow, but based on these reports such changes may be assumed to be present, especially in patients with advanced DR stages. Nevertheless, we did not find a difference in the outer retinal 
Table 3 Correlation of the retinal volume and thickness in relation to FAZ linear dimensions. $P$ values are given in the table

\begin{tabular}{lllllll}
\hline Linear dimensions $(\mu \mathrm{m})$ & & Horizontal & Vertical & Greatest & Smallest & Mean \\
\hline Total retinal thickness $(\mu \mathrm{m})$ & CMT & 0.346 & 0.476 & 0.391 & 0.528 & 0.381 \\
& paraS & 0.903 & 0.781 & 0.803 & 0.767 & 0.913 \\
& paraT & 0.254 & 0.182 & 0.320 & 0.249 & 0.215 \\
& paraI & 0.938 & 0.758 & 0.780 & 0.553 & 0.867 \\
& paraN & 0.736 & 0.705 & 0.744 & 0.698 & 0.883 \\
& periS & 0.583 & 0.806 & 0.951 & 0.618 & 0.702 \\
& periT & 0.217 & 0.261 & 0.288 & 0.258 & 0.228 \\
Outer retinal thickness $(\mu \mathrm{m})$ & periI & 0.922 & 0.630 & 0.469 & 0.944 & 0.761 \\
& periN & 0.542 & 0.925 & 0.456 & 0.835 & 0.728 \\
& periT & 0.862 & 0.572 & 0.903 & 0.653 & 0.825 \\
& paraT & 0.659 & 0.416 & 0.578 & 0.665 & 0.430 \\
Inner retinal thickness $(\mu \mathrm{m})$ & paraN & 0.684 & 0.321 & 0.550 & 0.258 & 0.497 \\
& periN & 0.240 & 0.457 & 0.230 & 0.487 & 0.224 \\
& periT & 0.926 & 0.885 & 0.797 & 0.767 & 0.974 \\
& paraT & 0.897 & 0.693 & 0.866 & 0.479 & 0.904 \\
& paraN & 0.890 & 0.480 & 0.851 & 0.802 & 0.673 \\
& periN & 0.409 & 0.410 & 0.449 & 0.317 & 0.342 \\
Ganglion cell layer thickness $(\mu \mathrm{m})$ & periT & 0.491 & 0.078 & 0.171 & 0.100 & 0.175 \\
& paraT & 0.312 & 0.682 & 0.625 & 0.764 & 0.428 \\
& paraN & 0.854 & 0.508 & 0.774 & 0.608 & 0.894 \\
& periN & 0.240 & 0.037 & 0.101 & 0.082 & 0.115 \\
& & 0.914 & 0.882 & 0.766 & 0.723 & 0.867 \\
\hline & & & & & &
\end{tabular}

$\mathrm{CMT}=$ central macular thickness; peri $=$ perifoveal; para $=$ parafoveal; $\mathrm{S}=$ superior; $\mathrm{T}=$ temporal; $\mathrm{I}=$ inferior; $\mathrm{N}=$ nasal previously reported findings, as no association between FAZ outline and ganglion cell layer thickness was found, most likely due to the low number of patients in whom we were able to identify the borders and measure the thickness.

We have not found an association between FAZ outline and retinal structural features such as: continuity of the external limiting membrane and inner segment/outer segment junction (within $500 \mu \mathrm{m}$ nasally and temporally from the fovea), and identification of a boundaries of the ganglion cell layer and between inner and outer retina. The latter was possible in almost every patient. It might be attributed to the dual circulation to the retina and the presence of the watershed zone at the outer plexiform layer. As suggested earlier, choroidal circulation supplying outer retina appeared to be sufficient in our patients, even in the face of reduced blood supply to the inner retina. Furthermore, continuity of the external limiting membrane and inner segment/outer segment junction, belonging to the outer retina, seems not to differ in association with FAZ outline damage for the same reason. In contrast, the identification of ganglion cell layer boundaries was significantly more difficult in patients with more severe FAZ outline damage (impossible in $29 \%$ in $\leq$ grade 2 versus $65 \%$ in $\geq$ grade $3, p=0.009)$. As the ganglion cell layer is supplied by the superficial capillary plexus, the damage of which is reflected in FA by FAZ outline destruction, the association appears to be direct. Yeung et al. showed that loss of retinal 
layers, more prominent within the inner retina, corresponded to areas of capillary non-perfusion on FA [18]; our results support this finding. In conclusion, based on our results, we believe that the loss of ganglion cell layer boundaries, and not necessarily the thickness, is associated with the progression of FAZ outline damage.

FAZ size and, in particular, outline constitute indicators of inner retina perfusion within the macula. The ischemic state of the macula corresponds to the destruction of the FAZ outline and related enlargement $[5,8,24]$. Our study confirmed the association between FAZ size and outline. Also, our results indicate that FAZ size does not correspond to retinal thickness or volume. This conclusion seems reasonable, as FAZ size is related to FAZ outline; the latter is not associated with retinal thickness or volume. Moreover, even in healthy subjects FAZ size varies widely [1]. Furthermore, Byeon et al. suggested that FAZ size does not represent the degree of damage to the inner retina [19].

Weaknesses are present within our study, including the retrospective character of research and limited number of patients. In order to select a particular group of patients (severe NPDR and PDR) and to meet the exclusion criteria, in particular the absence of CSME, fluorescein angiograms of 315 consecutive patients (almost 600 eyes) with any stage of DR were reviewed. However, only 51 eyes $(8.5 \%)$ were suitable for further evaluation.

The following points highlight the strengths of our investigation with regard to study design and material and methods:

1. Association between retinal thickness/volume and FAZ outline;

2. Focus on a particular group of patients (presenting with severe NPDR and PDR, without CSME), who are often excluded from other studies;

3. Subdivision of the retinal thickness in the inner and outer retina to investigate the influence of both blood supplies;

4. Measurement of ganglion cell layer thickness and assessment of its boundaries.

In the literature, contradictory data is presented on the role of the two imaging methods, OCT and FA, in the detection of ischemic diabetic maculopathy [16-19]. Our study did not provide a definitive answer to this issue. Nevertheless, at the present time it seems that OCT cannot replace AF in this regard. FA enables the evaluation of foveal perfusion, which, according to our study, could not be predicted based on OCT.

Acknowledgments Parts of this paper were presented at the German Ophthalmological Society Congress 19-22.09.2013 in Berlin and got the DOG Travel Award.

Conflict of interest No conflicts of interest to declare.
Open Access This article is distributed under the terms of the Creative Commons Attribution License which permits any use, distribution, and reproduction in any medium, provided the original author(s) and the source are credited.

\section{References}

1. Byeon SH, Kim M, Kwon OW (2012) Ischemia. In: Bernardes R, Cunha-Vaz J (eds) Optical coherence tomography. A clinical and technical update. Springer, Berlin, pp 24-26

2. Querques G, Lattanzio R, Querques L, Del Turco C, Forte R, Pierro L, Souied EH, Bandello F (2012) Enhanced depth imaging optical coherence tomography in type 2 diabetes. Invest Ophthalmol Vis Sci 53(10):6017-6024

3. Regatieri CV, Branchini L, Carmody J, Fujimoto JG, Duker JS (2012) Choroidal thickness in patients with diabetic retinopathy analyzed by spectral-domain optical coherence tomography. Retina 32(3):563-568

4. Vujosevic S, Martini F, Cavarzeran F, Pilotto E, Midena E (2012) Macular and peripapillary choroidal thickness in diabetic patients. Retina 32(9): 1781-1790

5. Bresnick GH, Condit R, Syrjala S, Palta M, Groo A, Korth K (1984) Abnormalities of the foveal avascular zone in diabetic retinopathy. Arch Ophthalmol 102(9):1286-1293

6. Arend O, Wolf S, Jung F, Bertram B, Pöstgens H, Toonen H, Reim M (1991) Retinal microcirculation in patients with diabetes mellitus: dynamic and morphological analysis of perifoveal capillary network. Br J Ophthalmol 75(9):514-518

7. Mansour AM, Schachat A, Bodiford G, Haymond R (1993) Foveal avascular zone in diabetes mellitus. Retina 13(2):125-128

8. Conrath J, Giorgi R, Raccah D, Ridings B (2005) Foveal avascular zone in diabetic retinopathy: quantitative vs qualitative assessment. Eye (Lond) 19(3):322-326

9. Hee MR, Puliafito CA, Duker JS, Reichel E, Coker JG, Wilkins JR, Schuman JS, Swanson EA, Fujimoto JG (1998) Topography of diabetic macular edema with optical coherence tomography. Ophthalmology 105(2):360-370

10. Schaudig UH, Glaefke C, Scholz F, Richard G (2000) Optical coherence tomography for retinal thickness measurement in diabetic patients without clinically significant macular edema. Ophthalmic Surg Lasers 31(3):182-186

11. Lattanzio R, Brancato R, Pierro L, Bandello F, Iaccher B, Fiore T, Maestranzi G (2002) Macular thickness measured by optical coherence tomography (OCT) in diabetic patients. Eur J Ophthalmol 12(6): 482-487

12. Sánchez-Tocino H, Alvarez-Vidal A, Maldonado MJ, MorenoMontañés J, García-Layana A (2002) Retinal thickness study with optical coherence tomography in patients with diabetes. Invest Ophthalmol Vis Sci 43(5):1588-1594

13. Sakata K, Funatsu H, Harino S, Noma H, Hori S (2007) Relationship of macular microcirculation and retinal thickness with visual acuity in diabetic macular edema. Ophthalmology 114(11):2061-2069

14. Soliman W, Sander B, Jørgensen TM (2007) Enhanced optical coherence patterns of diabetic macular oedema and their correlation with the pathophysiology. Acta Ophthalmol Scand 85(6):613-617

15. Soliman W, Sander B, Hasler PW, Larsen M (2008) Correlation between intraretinal changes in diabetic macular oedema seen in fluorescein angiography and optical coherence tomography. Acta Ophthalmol 86(1):34-39

16. Goebel W, Kretzchmar-Gross T (2002) Retinal thickness in diabetic retinopathy: a study using optical coherence tomography (OCT). Retina 22(6):759-767 
17. Ozdek SC, Erdinç MA, Gürelik G, Aydin B, Bahçeci U, Hasanreisoğlu B (2005) Optical coherence tomographic assessment of diabetic macular edema: comparison with fluorescein angiographic and clinical findings. Ophthalmologica 219(2):86-92

18. Yeung L, Lima VC, Garcia P, Landa G, Rosen RB (2009) Correlation between spectral domain optical coherence tomography findings and fluorescein angiography patterns in diabetic macular edema. Ophthalmology 116(6):1158-1167

19. Byeon SH, Chu YK, Lee H, Lee SY, Kwon OW (2009) Foveal ganglion cell layer damage in ischemic diabetic maculopathy: correlation of optical coherence tomographic and anatomic changes. Ophthalmology 116(10):1949.e1948-1959.e1948

20. van Dijk HW, Verbraak FD, Kok PH, Garvin MK, Sonka M, Lee K, Devries JH, Michels RP, van Velthoven ME, Schlingemann RO, Abràmoff MD (2010) Decreased retinal ganglion cell layer thickness in patients with type 1 diabetes. Invest Ophthalmol Vis Sci 51(7): 3660-3665

21. Early Treatment Diabetic Retinopathy Study research group (1985) Photocoagulation for diabetic macular edema. ETDRS report number 1. Arch Ophthalmol 103(12):1796-1806

22. Early Treatment Diabetic Retinopathy Study research group (1991) Fundus photographic risk factors for progression of diabetic retinopathy. ETDRS report number 12. Ophthalmology $98(5$ Suppl):823-833

23. Early Treatment Diabetic Retinopathy Study research group (1991) Grading diabetic retinopathy from stereoscopic color fundus photographs - an extension of the modified Airlie House classification. ETDRS report number 10. Ophthalmology 98(5 Suppl):786806

24. Early Treatment Diabetic Retinopathy Study research group (1991) Classification of diabetic retinopathy from fluorescein angiograms. ETDRS report number 11. Ophthalmology $98(5$ Suppl):807-822

25. Fine BS, Brucker AJ (1981) Macular edema and cystoid macular edema. Am J Ophthalmol 92(4):466-481

26. Yanoff M, Fine BS, Brucker AJ, Eagle RC (1984) Pathology of human cystoid macular edema. Surv Ophthalmol 28(Suppl): 505-511

27. Dimitrova G, Kato S, Tamaki Y, Yamashita H, Nagahara M, Sakurai M, Kitano S, Fukushima H (2001) Choroidal circulation in diabetic patients. Eye (Lond) 15(Pt 5):602-607

28. Nagaoka T, Kitaya N, Sugawara R, Yokota H, Mori F, Hikichi T, Fujio N, Yoshida A (2004) Alteration of choroidal circulation in the foveal region in patients with type 2 diabetes. Br J Ophthalmol 88(8): 1060-1063

29. Guyer DR, Schachat AP, Green WR (2009) The choroid: Structural considerations. In: Hinton DR, Schachat AP (eds) Retina. Basic science, inherited retinal disease, and tumors, vol 1. Elsevier, India, pp 33-36

30. Harris A, Bingaman D, Ciulla TA, Martin B (2009) Retinal and choroidal blood flow in health and disease. In: Hinton DR, Schachat AP (eds) Retina. Basic science, inherited retinal disease, and tumors, vol 1. Elsevier, India, p 89 